\title{
Des principes pratiques importants pour l'éthique clinique
}

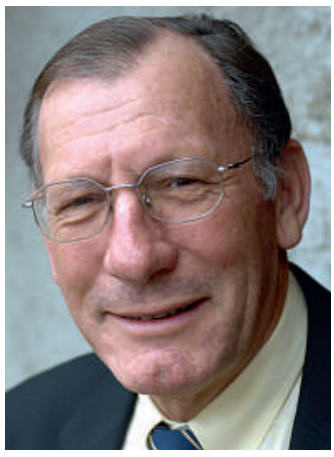

Jean Martin
L'ASSM a mis en consultation un projet de recommandations qui mérite de retenir l'attention [1]. La sous-commission qui l'a élaboré relève que les demandes de soutien éthique surviennent dans «une situation éthiquement difficile, exprimée par exemple par un malaise, un désaccord ou une incertitude sur comment bien faire...». Ce qui rappelle le titre judicieux d'un ouvrage de l'éthicien Eric Fuchs, qui a été un pionnier de la bioéthique en Suisse [2].

Une structure éthique contribue à la reconnaissance des conflits de valeurs et d'intérêts. Point majeur: ses avis sont consultatifs; leur seule autorité est la conviction emportée - ou non - par ses arguments. Le but n'est pas de retirer la responsabilité des professionnels mais de les accompagner au travers de dilemmes. La décision reste celle du médecin et de l'équipe soignante et le rôle qui doit être accordé à l'autonomie du patient reste inchangé (un second point majeur). Il est précisé aussi que les recommandations d'un comité d'éthique ne peuvent avoir le caractère de directives que si elles sont adoptées en tant que telles par l'instance légitimée à décider.

Le document décrit les diverses modalités possibles et souligne qu'il n'y a pas une seule manière de «bien faire», qu'il importe d'être pragmatique. «Le choix des structures doit refléter les tâches attribuées. A l'heure actuelle aucun modèle ne se détache comme supérieur aux autres.»

La sous-commission fait œuvre utile en s'efforçant de définir les qualités souhaitables de membres d'une structure éthique, dont elle souligne que la composition doit être multidisciplinaire. Eléments fondamentaux parmi ces qualités: «faire preuve d'introspection sur ses propres choix de valeur»; «être prêt à reconsidérer son avis initial à la lumière d'une discussion commune - et avec des personnes de points de vue différents»; «être prêt à acquérir une vision réaliste du quotidien clinique». Les membres doivent acquérir certaines compétences, dont: «savoir différencier la réflexion éthique du jugement moral»; «être conscient de la différence entre le normatif et le descriptif»; «être capable de distinguer l'éthique du droit et établir un lien entre les deux».

Quand un éthicien clinique intervient avec une équipe, il le fait en principe comme modérateur, aidant notamment les soignants à clarifier leurs valeurs. Dans ce cadre, il importe de réfléchir à l'inclusion du patient (comment et à quel point) et de ses proches dans le processus.
Aspect délicat: «L'utilité de structures éthiques repose à la fois sur leur indépendance et sur leur rattachement à l'institution. Elles doivent (...) se protéger de l'instrumentalisation.» Il convient que chaque institution précise qui chez elle peut saisir le service de soutien éthique et s'il y a des procédures particulières (à mon sens il faut être aussi souple que possible). Un dispositif est-il prévu pour les cas d'urgence? NB: les structures éthiques doivent pouvoir s'auto-saisir, à savoir décider de leur propre chef d'aborder un sujet. Une autre question est de savoir si l'avis éthique est diffusé, à qui et comment - en assurant bien sûr le respect du secret médical.

Dans une annexe, la sous-commission se penche sur le domaine de la médecine ambulatoire et des soins à domicile. «Les situations exigeant des décision éthiques n'y sont pas plus rares», liées notamment à des désaccords entre les personnes concernées dont le patient. Parmi les points à considérer, il y a le fait que les soins se déroulent au domicile privé: «Au quotidien, il arrive souvent que les normes personnelles divergent des normes professionnelles.» «Le patient entretient souvent des relations personnelles avec ses soignants. Celles-ci peuvent soit faciliter la prise de décisions éthiques, soit compliquer la reconnaissance des problèmes.» On peut noter que ces aspects sont comparables dans les homes pour personnes âgées ou dépendantes, qui deviennent aussi le domicile du pensionnaire.

L'administration peut-elle de même solliciter un conseil éthique? C'est en soi souhaitable mais la sous-commission note de possibles difficultés: «Une implication dans le soutien éthique à la direction peut faire surgir des conflits d'intérêts entre les facteurs économiques, institutionnels et politiques, et des dépendances qui doivent être réglées avec soin. La structure d'éthique doit avoir la possibilité de décliner des mandats sans avoir à en subir des conséquences négatives.»

L'importance de pouvoir faire appel à des mécanismes structurés de soutien éthique n'est plus mise en doute aujourd'hui et il est bon que l'ASSM émette des recommandations. Le projet mis en consultation aborde bien l'éventail des questions dont il faut tenir compte dans la pratique.

PS: la consultation sur le projet de l'ASSM court jusqu'à fin février. 